\title{
The far-ultraviolet main auroral emission at Jupiter - Part 2: Vertical emission profile
}

\author{
B. Bonfond ${ }^{1, *}$, J. Gustin ${ }^{1}$, J.-C. Gérard ${ }^{1}$, D. Grodent ${ }^{1}$, A. Radioti ${ }^{1}$, B. Palmaerts ${ }^{1,2}$, S. V. Badman ${ }^{3}$, K. K. Khurana ${ }^{4}$, \\ and C. Tao ${ }^{5}$ \\ ${ }^{1}$ Laboratoire de Physique Atmosphérique et Planétaire, Université de Liège, Allée du 6 Août, 19c, 4000 Liège, Belgium \\ ${ }^{2}$ Max-Planck-Institut für Sonnensystemforschung, Justus-von-Liebig-Weg 3, 37077 Göttingen, Germany \\ ${ }^{3}$ Lancaster University, Department of Physics, Lancaster, UK \\ ${ }^{4}$ University of California, Los Angeles, Los Angeles, California, USA \\ ${ }^{5}$ Institut de Recherche en Astrophysique et Planétologie, Toulouse, France \\ * Invited contribution by B. Bonfond, recipient of the EGU Division Outstanding Young Scientists Award 2015.
}

Correspondence to: B. Bonfond (b.bonfond@ulg.ac.be)

Received: 29 June 2015 - Revised: 10 September 2015 - Accepted: 17 September 2015 - Published: 1 October 2015

\begin{abstract}
The aurorae at Jupiter are made up of many different features associated with a variety of generation mechanisms. The main auroral emission, also known as the main oval, is the most prominent of them as it accounts for approximately half of the total power emitted by the aurorae in the ultraviolet range. The energy of the precipitating electrons is a crucial parameter to characterize the processes at play which give rise to these auroral emissions, and the altitude of the emissions directly depends on this energy. Here we make use of far-UV (FUV) images acquired with the Advanced Camera for Surveys on board the Hubble Space Telescope and spectra acquired with the Space Telescope Imaging Spectrograph to measure the vertical profile of the main emissions. The altitude of the brightness peak as seen above the limb is $\sim 400 \mathrm{~km}$, which is significantly higher than the $250 \mathrm{~km}$ measured in the post-dusk sector by Galileo in the visible domain. However, a detailed analysis of the effect of hydrocarbon absorption, including both simulations and FUV spectral observations, indicates that FUV apparent vertical profiles should be considered with caution, as these observations are not incompatible with an emission peak located at $250 \mathrm{~km}$. The analysis also calls for spectral observations to be carried out with an optimized geometry in order to remove observational ambiguities.
\end{abstract}

Keywords. Atmospheric composition and structure (airglow and aurora) - magnetospheric physics (auroral phenomena; current systems)

\section{Introduction}

The far-UV (FUV) aurorae at Jupiter can be divided into three major regions: (1) the outer emissions, including the satellite footprints, relatively compact blobs associated with injection signatures as well as more diffuse emissions associated with the pitch angle diffusion boundary; (2) the main emission, an essentially closed auroral curtain associated with corotation enforcement electric currents; and (3) the polar emissions, which can themselves be divided into (a) an active region, the locus of very intense flares, (b) a chaotic and dynamic swirl region, and (c) a dark region, which may sometimes be flanked by polar dawn spots (see reviews by Grodent, 2015; Delamere et al., 2014, and references therein). Previous observations of the FUV main emission mainly focussed on its location (Grodent et al., 2003, 2008; Bonfond et al., 2012; Vogt et al., 2011), on its brightness' spatial (Radioti et al., 2008; Palmaerts et al., 2015; Bonfond et al., 2015) or temporal (Grodent et al., 2003; Nichols et al., 2007, 2009) variations, as well as on the spatial and temporal variability in absorption spectra (Gustin et al., 2004, 2006; Gérard et al., 2014). The present study focusses on the vertical brightness profile as seen above the limb of the planet by the Hubble Space Telescope's far-UV instruments.

According to Knight's theory of field-aligned currents (Knight, 1973; Lundin and Sandahl, 1978), the precipitating energy flux and the electron energy should be correlated. Moreover, the vertical extent of auroral emissions directly 
depends on the energy of the precipitating charged particles: the more energetic, the deeper they release their energy to the background atmospheric particles and the deeper the subsequent light emissions. There are two ways to estimate the altitude of the auroral emissions at Jupiter. The most common but somewhat indirect one is based on the so-called color ratio. As the methane density decreases sharply above the homopause (around $350 \mathrm{~km}$ above the $1 \mathrm{bar}$ level, considered as the reference level hereafter), the ratio of unabsorbed emissions over absorbed emission is indicative of the atmospheric depth at which the emission took place (Yung et al., 1982). This FUV color ratio is usually defined as the ratio of $\mathrm{H}_{2}$ emissions from 155 to $162 \mathrm{~nm}$ over $\mathrm{H}_{2}$ emissions from 123 to $130 \mathrm{~nm}$, both expressed in $\mathrm{kR}$. Assumptions on the energy distribution of the precipitating electrons and on the vertical distribution of methane in the atmosphere allow the conversion from the color ratio to the energy of the precipitating electrons. Based on color ratio measurements in the main emission, Gustin et al. (2004) showed that the precipitating energy flux, which ranges between $\sim 2$ and $\sim 30 \mathrm{~mW} \mathrm{~m}^{-2}$, is indeed positively correlated with the electron mean energy, which ranges from $\sim 30$ to $\sim 200 \mathrm{keV}$ on the dayside.

The second method consists of directly measuring the vertical profile of the emission above the limb. However, such a measurement requires a good understanding of the location of the limb and an observing geometry such that auroral emission lies in the limb plane rather than in front of it or behind it. So far, the most accurate measurements of the altitude of the main emission have been derived from five images of the post-dusk sector in the visible wavelength by Galileo's Solid State Imaging (SSI) system (Vasavada et al., 1999). The altitude of the brightness peak of these emissions was measured to be $250 \mathrm{~km}$ above the limb of the planet. Similar to FUV auroral emissions, such visible emissions arise from the deexcitation of $\mathrm{H}_{2}$ molecules and $\mathrm{H}$ atoms (James et al., 1998). More specifically, these emissions mainly consist of continuum emission from triplet states of $\mathrm{H}_{2}$ and Balmer series lines of $\mathrm{H}$ following the dissociative excitation of $\mathrm{H}_{2}$. Hence the visible vertical emission profile is expected to be very similar to the one observed in the FUV. Similar measurements, but with a much lower spatial resolution, have been performed in the infrared $\mathrm{K}$ band from the Subaru telescope (Uno et al., 2014). The peak altitude determined for the $\mathrm{H}_{2}$ quadrupole emissions, which result from processes similar to those leading to the visible and UV emissions, is much higher: 590-720 km. Hubble Space Telescope (HST) FUV images regularly display portions of the main emission above the planetary limb, making it possible to directly measure its vertical emission profile on the nightside. Cohen and Clarke (2011) found some significant hemispheric differences in the scale height of the FUV emissions at a high altitude above the limb, which they attributed to temperature differences between the two polar regions. But they did not examine the peak altitude of the emissions, which reflects the energy of the precipitating electrons. The statistical analysis carried out in Sect. 2.1 shows that the apparent peak altitude on the nightside is significantly higher than the one from the Galileo observations. However, we show in Sect. 2.2 that a good understanding of the impact of hydrocarbon (and more specifically methane) absorption is necessary before we can draw firm conclusions from this apparent FUV peak altitude.

\section{Observations of the nightside main emission above the limb}

\subsection{Imaging observations of the vertical profile}

The extraction of the vertical brightness profiles above the limb requires not only a high spatial resolution but also the precise determination of the $0 \mathrm{~km}$ reference level, assigned to the 1 bar level. For this reason, we use the large data set of 1663 images acquired with the Solar Blind Channel (SBC) of the Hubble Space Telescope's Advanced Camera for Surveys (ACS). This channel is dedicated to the far UV in the range from 115 to $170 \mathrm{~nm}$ and has a plate scale of 0.032 arcsec pixel $^{-1}$. Two filters have been used to observe Jupiter's aurorae: the F115LP filter, which includes the H Lyman- $\alpha$ line, and the F125LP, which does not. ACS-SBC is known to be affected by some "red leak", which allows a significant amount of near-UV and visible light into the instrument (Boffi et al., 2008). In our images, these photons hitting the detector result from the Rayleigh scattering of the solar light by Jupiter's neutral atmosphere. As a consequence, the planetary limb is particularly crisp in the images, as it corresponds to the visible limb at the 1 bar level. Hence, this originally unwanted solar reflection actually constitutes a valuable reference to estimate the altitude of auroral emissions above the limb (see discussion in Bonfond et al., 2009).

In order to measure the altitude of the main emission in ACS images, we follow the procedure described by Bonfond et al. (2009). We extract successive cuts across the auroral emissions in such a way that each profile intercepts the planetary center. The scan angle is defined so that $0^{\circ}$ corresponds to the dawn equator and $90^{\circ}$ corresponds to the North Pole. The range of the scan is defined manually in order to only cover the main emission and avoid footprint emissions. For each cut, a vertical profile is extracted and fitted by the sum of two decreasing exponential functions and a Chapman profile of the form

$f(Z)=C \exp \left(1-\frac{Z-Z_{0}}{H}-\exp \left(-\frac{Z-Z_{0}}{H}\right)\right)$,

where $C$ is a constant, $Z$ is the altitude, $Z_{0}$ is the altitude of the peak, and $H$ is the scale height. The two exponential functions account for the combination of (a) the blurring of the planetary disk by the instrumental point spread function, (b) the molecular and atomic hydrogen airglow, and (c) the extended thermospheric emissions discussed by Cohen and Clarke (2011). Such a combination of exponential functions 
has proved to fit the observed residual vertical profile right outside the auroral region adequately. We then subtract the sum of the two exponentials from the observed vertical profile and determine the altitude of the emission peak. In order to avoid signal-to-noise ratio problems, the profiles with a brightness at the peak altitude below one third of the brightness at the $0 \mathrm{~km}$ altitude on the original profile are discarded. Profiles containing pixels in the bad row of the detector (i.e., a row of non-functioning pixels) are also discarded. Figure 1 (top panel) shows an ACS image of the northern aurorae on which the main emission is clearly seen above the limb. The middle panel shows the evolution of the peak altitude as a function of the scan angle. In this plot, the altitude of the discarded profiles is set to $0 \mathrm{~km}$. The bottom panel shows the initial observed vertical profile (in black), the sum of the best fit exponential functions in blue and the final vertical profile in red.

If the real peak altitude of the auroral curtain is uniform and if hydrocarbon absorption is negligible, then geometry implies that the apparent peak altitude as seen from HST reaches a maximum in the limb plane. The corresponding observed vertical profile is thus the real vertical profile and the corresponding peak altitude is the real one as well. For each sequence of ACS observations (also known as HST orbits), two images are selected: one with the F115LP filter and one with the F125LP filter. For sequences during which only one filter was used, only one image is selected. Figure 2 shows the peak altitude as a function of the central meridian longitude (CML) for all the images. With a typical peak altitude of $400 \pm 20 \mathrm{~km}$ in the nightside (for a $95 \%$ confidence level), our results are significantly different from the $250 \mathrm{~km}$ obtained by Galileo-SSI in the post-dusk side. Table 1 shows the means and the standard deviations of this measured maximum peak altitude according to hemisphere and filter. No clear discrepancy is found whatever the filter or the hemisphere under consideration.

We followed the same procedure as Bonfond et al. (2009) to model the observed vertical profile using the Monte Carlo numerical model of electron transport from Shematovich et al. (2008) and the pressure-temperature relationship derived from the auroral atmosphere profile from Grodent et al. (2001). Figure 3 and Table 2 show that either a monoenergetic or a Maxwellian distribution with a mean energy of $\sim 30 \mathrm{keV}$ already provides convincing fits of the observed profile when they are convolved with the instrumental point spread function. A Kappa energy distribution could also be used to fit the profile, but this added level of complexity only marginally improves the quality of the fit. The mean energy we obtain (around $20-35 \mathrm{keV}$ ) is at the lowest end of the range of previous estimates of the electron energy for the main emission based on the color ratio method (i.e., between 30 and $200 \mathrm{keV}$ ) (Gustin et al., 2004; Gérard et al., 2014). It is nevertheless noteworthy that the vertical distribution of hydrocarbons on the polar atmosphere of Jupiter is poorly constrained by direct observations and that different sets of
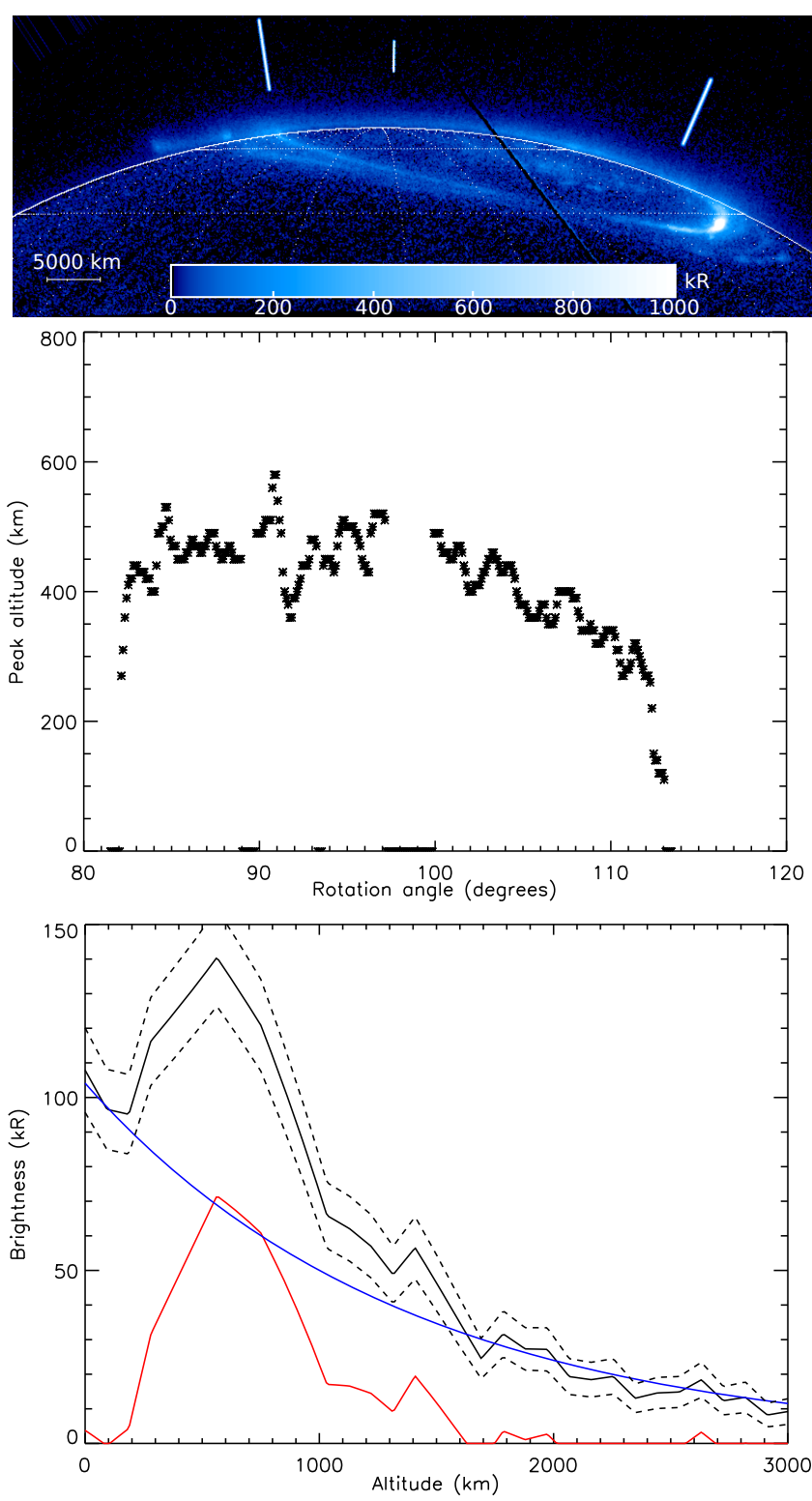

Figure 1. Top panel: example of an ACS image, acquired with the F125LP filter on 6 June 2007. The main emission is clearly seen above the limb. The two long lines indicate the limits of the scan and the small one indicates the location of the selected profile. Middle panel: profile of the peak altitude as a function of the rotation scan angle (stars); $0^{\circ}$ corresponds to the dawn equator and $90^{\circ}$ corresponds to the North Pole. Bottom panel: example of an extracted vertical profile (solid black line), along with the associated uncertainty (dashed lines). The blue line represent the best fit background profile and the red line represents the resulting vertical auroral profile.

hypotheses lead to somewhat different values (Gérard et al., 2014). Should the actual methane homopause be located at a higher altitude than assumed by the models used to derive energies from the color ratios, then the results provided by this method would overestimate the actual electron energies. 


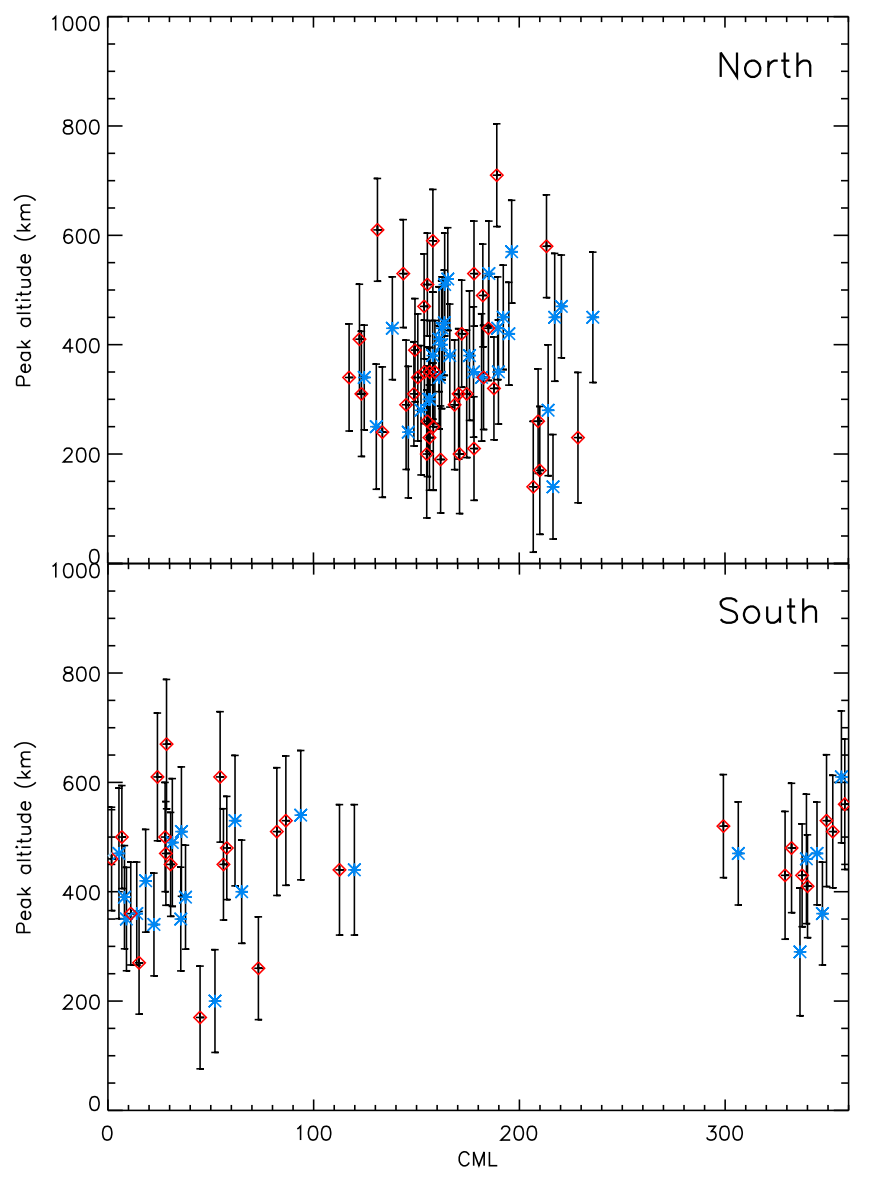

Figure 2. Plots of the altitude of the brightness peak of the main emission above the limb for the northern (top) and southern (bottom) hemispheres. Observations acquired with the F125LP filter are noted with blue stars and observations acquired with the F115LP filter are noted with red diamonds. The error bars represent the uncertainty regarding the altitude due to the uncertainty regarding the planetary center location.

Table 1. This table lists the mean and standard deviation of the measured altitude of the brightness peak in the main auroral emissions as observed above the limb for both hemispheres and filters. The uncertainty regarding the mean altitude represents the $95 \%$ confidence interval and is computed as twice the standard error of the mean $\sigma_{\mathrm{m}}=\frac{\sigma}{\sqrt{N}}$, with $N$ being the number of observations.

\begin{tabular}{ccc}
\hline & North & South \\
\hline & \multicolumn{2}{c}{ Mean of the peak altitude } \\
\hline F115LP & $385 \pm 35 \mathrm{~km}$ & $421 \pm 41 \mathrm{~km}$ \\
F125LP & $354 \pm 44 \mathrm{~km}$ & $464 \pm 44 \mathrm{~km}$ \\
\hline & \multicolumn{2}{c}{ Standard deviation $(\sigma)$} \\
\hline F115LP & $95 \mathrm{~km}$ & $94 \mathrm{~km}$ \\
F125LP & $137 \mathrm{~km}$ & $111 \mathrm{~km}$ \\
\hline
\end{tabular}

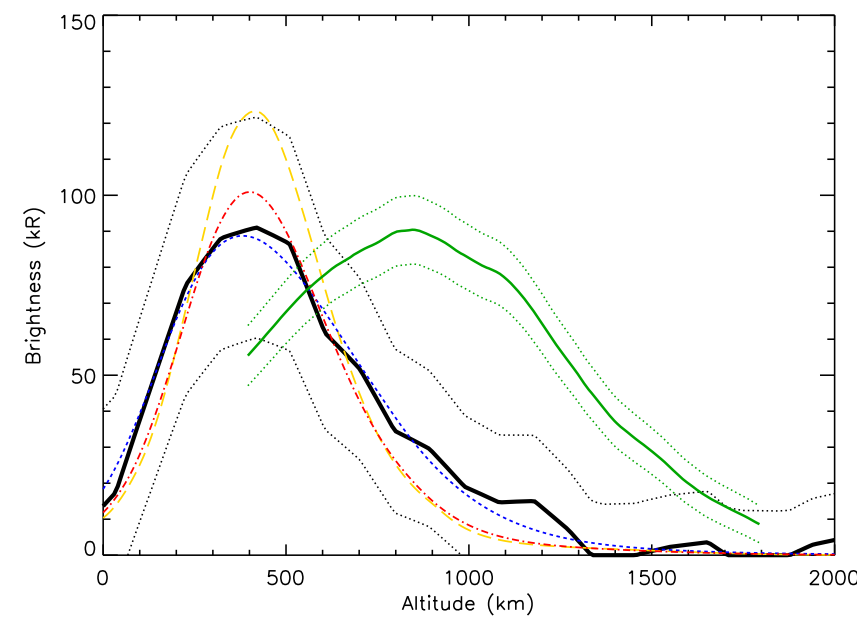

Figure 3. Best fits of a typical observed vertical profile after planetary disk subtraction (solid line), using a monoenergetic distribution (long yellow dashes), a Maxwellian distribution (red dash-dotted line), and a kappa distribution (blue short dashed line). The best fit parameters are listed in Table 2 . The measurement uncertainty is noted with the dotted black lines. The green curves shows the scaled Io footprint tail vertical profile from Bonfond et al. (2009) and their related uncertainties for comparison.

Table 2. Parameters of the best fit curves compared to the summed profile. The mean energy is computed as the ratio of the energy flux $\int_{0}^{\infty} E I(E) \mathrm{d} E$ over the particle flux $\int_{0}^{\infty} I(E) \mathrm{d} E$. Note that the kappa distribution is truncated at $328 \mathrm{keV}$.

\begin{tabular}{lrrr}
\hline Distribution & $\begin{array}{r}\text { Characteristic } \\
\text { energy }\left(E_{0}\right)\end{array}$ & $\begin{array}{r}\text { Spectral index } \\
(\kappa)\end{array}$ & $\begin{array}{r}\text { Mean } \\
\text { energy }\end{array}$ \\
\hline Monoenergetic & $36 \mathrm{keV}$ & - & $36 \mathrm{keV}$ \\
Maxwellian & $15 \mathrm{keV}$ & - & $31 \mathrm{keV}$ \\
Kappa & $2 \mathrm{keV}$ & 2.45 & $19 \mathrm{keV}$ \\
\hline
\end{tabular}

Contrary to the color ratio method, the limb measurement method is basically the same for both the Galileo-SSI images and the present HST-ACS images, as it consists of measuring the maximum vertical shift between the limb and the brightness peak. Hence, there are two possibilities. The first one is that the discrepancy in the altitude of the main emission between the FUV and the visible peak altitudes is real. Assuming that the five Galileo-SSI measurements are typical, this would indicate that the energy of the precipitating electrons is larger on the dusk side than on the nightside. The second possibility is that the apparent FUV vertical profiles observed by HST are affected by the hydrocarbon absorption at low altitude. This absorption at the bottom of the profile would artificially raise the observed peak altitude above the methane homopause.

A simulation of this effect, using the auroral atmosphere profile from Grodent et al. (2001), is shown in Fig. 4a. This simulation assumes that the main emission seen above the 
limb is a thin curtain in the limb plane and uses a typical auroral spectrum made of $\mathrm{H}_{2}$ Werner and Lyman bands. It then accounts for the wavelength-dependent absorption along the line of sight as a function of the altitude above the limb to compute the apparent vertical profile based on a prescribed emission profile. The two main FUV absorbing molecules in the Jovian atmosphere are $\mathrm{CH}_{4}$, with a cross section that abruptly rises below $140 \mathrm{~nm}$, and $\mathrm{C}_{2} \mathrm{H}_{2}$, which displays a much more complex cross section across the whole FUV domain. The left panel of Fig. 4a shows two realistic prescribed emissions profiles peaking at 250 and $400 \mathrm{~km}$. Apparent profiles, i.e., the profiles seen by an Earth-based observer after absorption along the line of sight, are shown in the middle and the right panels. The absorption is wavelength dependent and, for the sake of the example, these profiles account for the throughput of the F125LP filter of the ACS camera. The right panel curves also account for the instrumental point spread function (PSF). It can be seen that, because of the strong absorption by $\mathrm{CH}_{4}$ and $\mathrm{C}_{2} \mathrm{H}_{2}$ molecules at low altitude, the two apparent profiles end up to be very similar, especially after convolution by the PSF. As a conclusion, our model shows that the apparent vertical profile deduced from the FUV images alone cannot allow us to derive the vertical emission profile for the nightside main emission because the apparent peak altitude is too close to the methane homopause (acknowledging that the ACS-SBC plate scale typically corresponds to $\sim 130 \mathrm{~km}$ at the distance of Jupiter). Indeed, if the emission peak was located much higher, then only the bottom part of the apparent vertical profile would be attenuated, but the location of the brightness peak altitude would remain unchanged.

\subsection{Far-UV spectra above the limb}

One possible way to resolve the ambiguity raised in the previous section would be to search for absorption signatures in spatially resolved FUV spectra, such as those obtained with the G140L grating of the Space Telescope Imaging Spectrograph (STIS). This observing mode offers spatially resolved spectroscopy in the range from 115 to $170 \mathrm{~nm}$. The slit length covers 25 arcsec and the width used to observe Jupiter in the FUV is either 0.2 or 0.5 arcsec. Although it is expected that observations of a light source at slant angles accumulate more absorbing molecules along the line of sight, the spectral observations of the auroral emissions at the limb usually bear less absorption signatures than those located closer to the center of the planetary disk (e.g., Gustin et al., 2004; Gérard et al., 2014). These weak absorption signatures observed above the limb suggests that the nightside main emission actually takes place at relatively high altitude. However, the representativeness of the few spectra found in the literature is not clear. Hence, in the present study, we revisit the whole observation set gathered from 1997 to 2014 with STIS.

Our study requires the selection of spectra capturing the main emission above the limb. This criterion rules out spec-
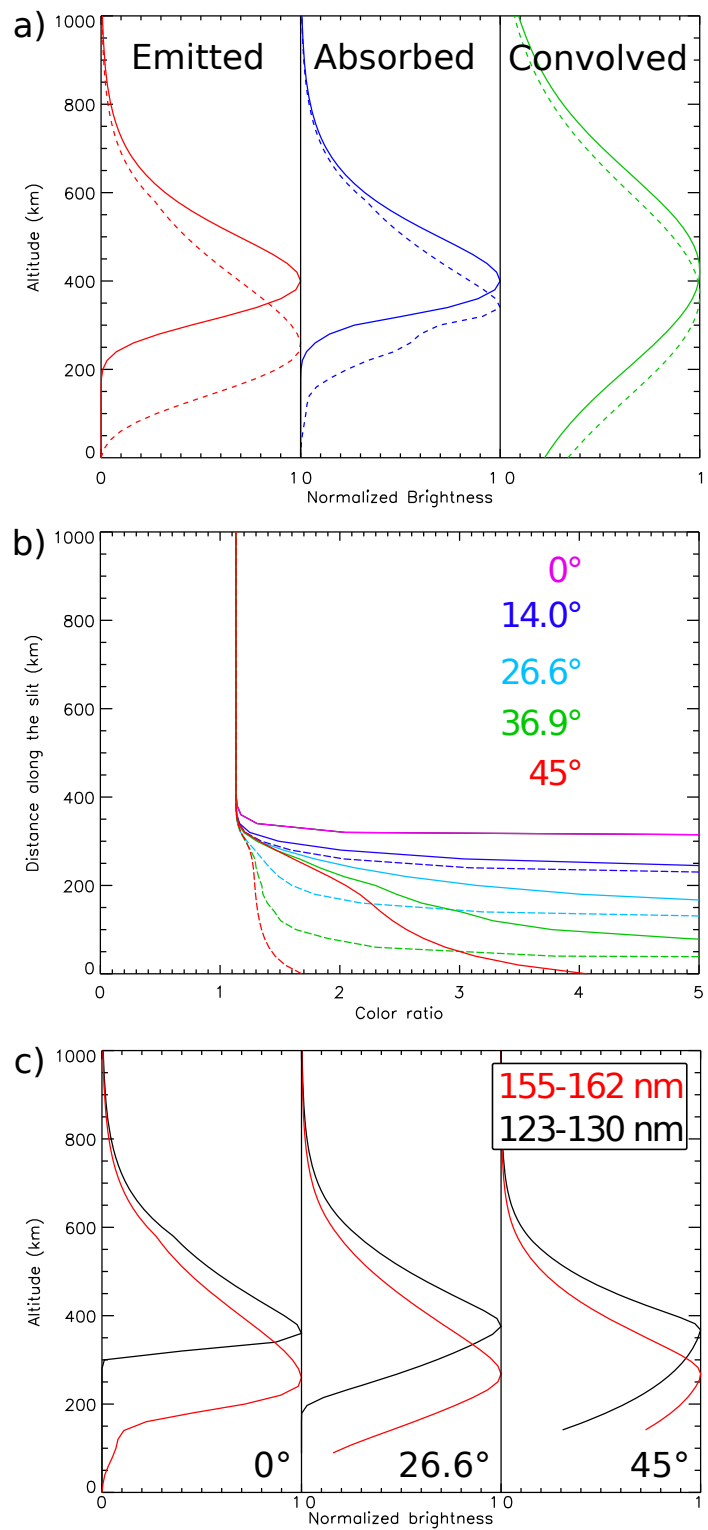

Figure 4. Panel (a) shows the $\mathrm{UV} \mathrm{H}_{2}$ emission vertical profile, the apparent profile as seen by ACS/SBC with the F125LP filter above the limb, and the convolved apparent profiles for two test profiles. The first one peaks at $400 \mathrm{~km}$ (solid line) and the second one peaks at $250 \mathrm{~km}$ (dashed line). Panel (b) shows the apparent color ratios for various angles between the 0.5 arcsec slit and the normal to the limb for two simulated vertical profiles peaking at $400 \mathrm{~km}$ (solid lines) and $250 \mathrm{~km}$ (dashed lines). Panel (c) shows the apparent profiles in the $155-162 \mathrm{~nm}$ range (red) and $123-130 \mathrm{~nm}$ range (black) for an emissions profile peaking $250 \mathrm{~km}$ above the limb.

tra intersecting the main emission at an ansa or not intersecting the main emission at all. The location of the slit of the spectrograph relative to the aurora cannot be deduced from the spectral data alone because of the pointing uncertainties. Accordingly, we only use spectra for which a context auroral image was acquired a few minutes before or after the spec- 


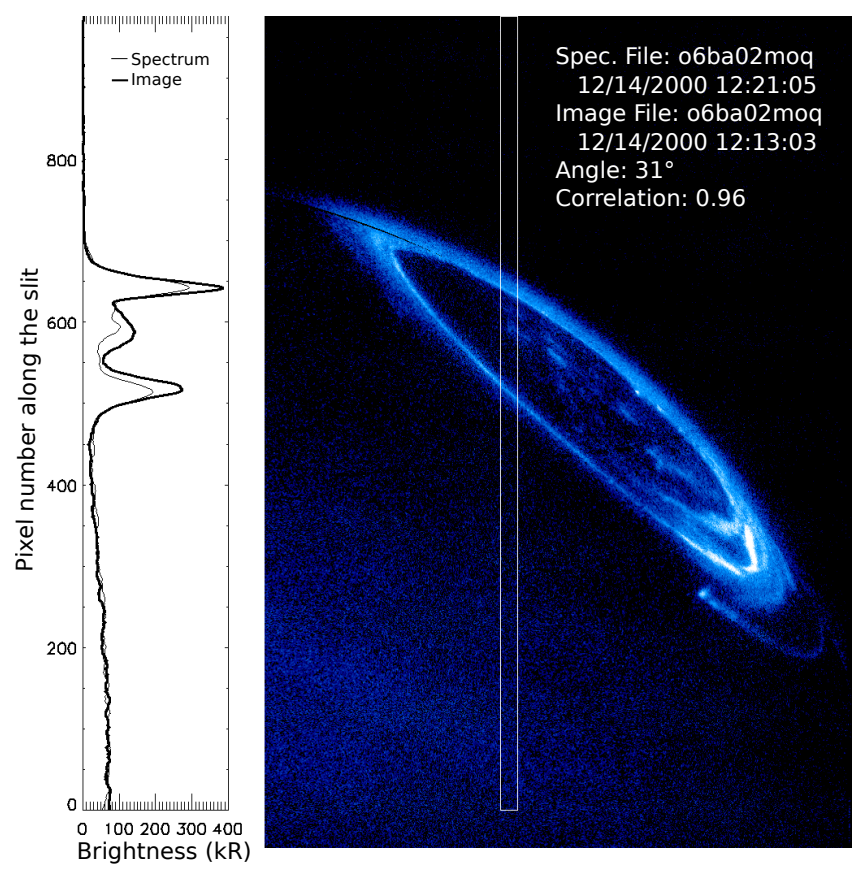

Figure 5. Example of a reconstructed STIS image corresponding to a spectral observation. The white rectangle represents the location of the slit. The spectral profile (thick line) versus the pixel number is shown on the side plot and the best fit profile from the reconstructed image is shown by the thin line. This image clearly shows that the slit is not normal to the limb and that any point on a profile along the slit mixes emissions from different altitudes.

tral exposure. In order to account for the time delay between the imaging and the spectral information, cylindrical projections of these context images are back-projected accounting for the planetary rotation (see Fig. 5). Brightness profiles extracted from the reconstructed images are then compared to the brightness profile along the slit in order to retrieve the location of the slit relative to the aurora. The localization of the slit position also allows the computation of the angle between the slit and the normal to the local limb. It should, however, be noted that the correspondence between the profiles is not always very good because of the temporal variability in the polar emissions. As a consequence, we estimate the uncertainty regarding the determination of the angle to lie around a few degrees.

Unfortunately, no HST spectral observation has been carried out with the slit exactly perpendicular to the limb (see Table 3). When we include the $\mathrm{C}_{2} \mathrm{H}_{2}$ absorption in our model, we note that even emissions at wavelength longer than $140 \mathrm{~nm}$ are significantly absorbed. Because of the slit tilt angle and the finite slit width, the brightness at a specific point along the slit is the result of the accumulation of light from different altitudes along the line of sight (see Fig. 5). Emissions from low altitude, which have a high color ratio but are dim because they are strongly absorbed by $\mathrm{C}_{2} \mathrm{H}_{2}$, are thus mixed with less absorbed emissions orig-
Table 3. List of the STIS G140L spectral observation used to determine the shift between the $\mathrm{H}_{2}$ brightness peak locations in the 123 $130 \mathrm{~nm}$ range and the $155-162 \mathrm{~nm}$ range. The angles between the slit and the normal to the local limb are noted in the third column. The fourth column indicates the shift between the unabsorbed and the absorbed brightness peaks as measured in pixels in the spectra. Finally, the fifth column displays a conversion of these shifts into kilometers, accounting for the cosine of the angle. Dates are given in the format of $\mathrm{mm} / \mathrm{dd} / \mathrm{yyyy}$.

\begin{tabular}{llrrr}
\hline Date & $\begin{array}{l}\text { File } \\
\text { rootname }\end{array}$ & Angle & $\begin{array}{r}\text { Shift } \\
\text { in pixels }\end{array}$ & $\begin{array}{r}\text { Vertical } \\
\text { shift }\end{array}$ \\
\hline 07/26/1998 16:01:22 & o43b2txmq & $17^{\circ}$ & 2 & $147 \mathrm{~km}$ \\
11/26/1998 02:17:03 & o43b14siq & $24^{\circ}$ & 1 & $75 \mathrm{~km}$ \\
08/15/1999 17:44:11 & o5g2e3d1q & $34^{\circ}$ & 2 & $135 \mathrm{~km}$ \\
02/22/2000 16:32:48 & o5hyb6g1q & $20^{\circ}$ & 2 & $181 \mathrm{~km}$ \\
11/14/2000 06:06:50 & o5hyb5ekq & $7^{\circ}$ & 0 & $0 \mathrm{~km}$ \\
11/14/2000 06:14:08 & o5hyb5emq & $8^{\circ}$ & 0 & $0 \mathrm{~km}$ \\
12/14/2000 12:21:05 & o6ba02moq & $31^{\circ}$ & 3 & $187 \mathrm{~km}$ \\
12/16/2000 12:34:55 & o6ba03v0q & $37^{\circ}$ & 1 & $58 \mathrm{~km}$ \\
01/01/2014 03:23:25 & oc1z011sq & $12^{\circ}$ & 1 & $73 \mathrm{~km}$ \\
01/03/2014 04:47:58 & oc1z03y8q & $7^{\circ}$ & 1 & $74 \mathrm{~km}$ \\
01/04/2014 01:31:02 & oc1z04b9q & $9^{\circ}$ & 1 & $74 \mathrm{~km}$ \\
01/05/2014 06:12:32 & oc1z05heq & $7^{\circ}$ & 2 & $148 \mathrm{~km}$ \\
01/06/2014 02:55:50 & oc1z06b2q & $20^{\circ}$ & 2 & $141 \mathrm{~km}$ \\
01/13/2014 22:59:53 & oc1z08ehq & $20^{\circ}$ & 0 & $0 \mathrm{~km}$ \\
01/16/2014 00:24:05 & oc1z12moq & $19^{\circ}$ & 1 & $71 \mathrm{~km}$ \\
\hline
\end{tabular}

inating from a higher altitude. The contamination by higheraltitude emissions can be such that the total absorption signature is strongly dampened. Figure $4 \mathrm{~b}$ shows the comparison between the color ratio profile for a profile peaking at 400 and $250 \mathrm{~km}$ for five slit tilt angles ranging from 0 to $45^{\circ}$. At $0^{\circ}$ the color ratio profile is the same for the two emission profiles, and it rises steeply around $350 \mathrm{~km}$. As the angle increases, the color ratio profiles become increasingly different and they increase much less sharply with decreasing altitudes. This geometrical effect could explain the relatively low values observed at the limb for previously published color ratios. Moreover, in practice, uncertainties regarding the slit angle, the impact of the point spread function, and contamination by nonauroral emissions (such as airglow emissions or solar light reflection) at the bottom of the profiles (i.e., below $200 \mathrm{~km}$ ) result in ambiguities concerning the actual amount of absorption.

Fortunately, another parameter may provide more robust evidence of methane absorption in the spectral observations: the shift between the apparent brightness in the $123-130 \mathrm{~nm}$ range (absorbed by $\mathrm{CH}_{4}$ ) and the 155-162 nm range (not absorbed by $\mathrm{CH}_{4}$ ). Figure $4 \mathrm{c}$ shows the simulated observable brightness profile for these two wavelengths, assuming the same emitted profile peaking at $250 \mathrm{~km}$ as in Fig. $4 \mathrm{a}$, for slit angles of $0,26.6$, and $45^{\circ}$. Although the increasing angle results in a smoothing of the pair of curves and a shift in the peak locations, the relative location of the peaks slightly increases as the secant of the angle. Nevertheless, at larger 
angles, the smoothing of the profiles will make the identification of the peaks increasingly problematic in practice, due to the limited signal-to-noise ratio in the spectra. Thus, in Table 3, which summarizes our results, observations at angles larger than $45^{\circ}$ are excluded. The shift between the two observed peaks ranges between 0 and 3 pixels, which corresponds to vertical distances of 0 to $185 \mathrm{~km}$ with an average value of $\sim 90 \mathrm{~km}$. Individual results should be considered carefully because of the limited signal-to-noise ratio. But the fact that the shift is systematically greater than or equal to $0 \mathrm{~km}$ and the relatively low dispersion of the results indicate that the mean value is robust. According to our simulations, a $90 \mathrm{~km}$ shift would be compatible with a brightness peak at $250 \mathrm{~km}$.

As a conclusion, absorption signatures at the limb are actually clearly distinguishable in the form of a shift between the absorbed and unabsorbed brightness profiles. This suggests that the preliminary results from the section above concerning the apparent vertical profile of the main emission could overestimate the peak altitude and thus underestimate the mean energy of the precipitating electrons. The effect of absorption could even reconcile the Galileo-SSI measurements and the HST-ACS measurements. It should, however, be noted that the altitude measurements are made in the limb plane, while the spectra are acquired at a random location on the nightside. Should the main emission auroral curtain be located behind the limb plane, which is likely, then it would be possible for emissions actually peaking at $400 \mathrm{~km}$ to display both an apparent peak at $400 \mathrm{~km}$ and some significant absorption. Hence, present data does not allow us to reach a firm conclusion on that matter. It would require a spectral scan with the slit as perpendicular as possible to the limb to verify whether the peak shift between absorbed and unabsorbed emissions persists all along the limb.

\section{Discussion and conclusions}

Observational evidence indicates that the current density, the precipitated energy flux, and the electron energy are correlated (Gustin et al., 2004). Moreover, as more energetic particles deposit their energy deeper into the atmosphere, the measurement of the auroral vertical profile reflects the energy distribution of the precipitating particles.

The HST image processing method used for estimating the altitude of the Io footprint at Jupiter (Bonfond et al., 2009) and the main oval emission at Saturn (Gérard et al., 2009) is used here for measuring the altitude of the Jovian main emission. The apparent peak altitude of the main emission above the limb is $\sim 400 \mathrm{~km}$, which clearly differs from the altitude of the Io footprint tail, which peaks at $900 \mathrm{~km}$ and exhibits a wider spread (see the green profile in Fig. 2). Such discrepancies most probably reveal a different acceleration mechanism as the main emission is associated with the buildup of a field-aligned potential leading to a narrow energy dis- tribution (e.g., Ray et al., 2012). On the other hand, the Io footprint is thought to stem from electron acceleration by inertial Alfvén waves leading to a broader energy dispersion (Hess et al., 2013). While a kappa distribution was necessary to fit the observed profile of the Io footprint, a monoenergetic or a Maxwellian energy distribution with a mean energy of $\sim 30 \mathrm{keV}$ would suffice to explain the main emission observations. Such a value is at the lower end of the $30-200 \mathrm{keV}$ energy range derived from the color ratio on the dayside (Gustin et al., 2004).

More importantly, the peak altitude measured in the FUV domain on the nightside $(400 \mathrm{~km})$ is significantly higher that the peak altitude measured in visible light on the post-dusk side $(250 \mathrm{~km})$ but lower than the altitude observed in the infrared for the $\mathrm{H}_{2}$ quadrupolar emissions (590-720 km). The vertical profiles in these three wavelength domains are expected to peak at similar altitudes because these emissions are largely dominated by electron impact excitation, mostly with secondary electrons. It is thus the altitude at which these secondary electrons are formed that controls the emission profile, whatever the wavelength. This is particularly true for the FUV emissions, which are essentially related to $\mathrm{H}_{2}$ Werner and Lyman bands emissions and H Lyman- $\alpha$ line emissions. It is also true for the mid-UV to visible range, which is related to the combination of (1) $\mathrm{H}_{2} \mathrm{~A} \rightarrow \mathrm{B}$ continuum emissions, (2) of strong lines related to the radiative decay of gerade singlet states of $\mathrm{H}_{2}$ and (3) of members of the $\mathrm{H}$ Balmer series resulting from the dissociative excitation of $\mathrm{H}_{2}$ (James et al., 1998). The case of the $\mathrm{H}_{2}$ quadrupole emissions is actually more complicated. First of all, $\mathrm{H}_{2}$ in the ground electronic state is not only vibrationally excited by direct electron impact and by excitation of the $\mathrm{H}_{2}$ Lyman and Werner bands but also by the dissociative recombination of $\mathrm{H}_{3}^{+}$(Cravens, 1987). Assuming that the electron impact excitation process is dominant, one would expect the excitation profiles for all three wavelengths to be fairly similar, but the lifetime of this vibrational excitation of $\mathrm{H}_{2}$ is relatively long ( $\sim$ a few tenths of a second; Uno et al., 2014). As a consequence, the collisional deactivation would increasingly supersede the radiative deactivation as the pressure increased. Radiative deexcitation would thus be very unlikely at a low altitude. Both the excitation by the dissociative recombination of $\mathrm{H}_{3}^{+}$and the collisional deexcitation would conspire to make the $\mathrm{H}_{2}$ quadrupole emission vertical profile peak at a higher altitude than the visible and UV emission profiles. Further theoretical analyses of the processes associated with the visible and the $\mathrm{H}_{2}$ quadrupole emissions as a function of the energy of the precipitating electrons could turn out to be very useful to quantitatively explain the observed discrepancy between the different wavelengths.

Should such a study confirm the expected similarity of the FUV and visible vertical emission profiles and should appropriate spectral observations show that the FUV emissions actually peak at $400 \mathrm{~km}$, then the discrepancy between the FUV peak altitude and the visible peak altitude may be interpreted 
as an indication that the nightside field-aligned currents are lower than the dusk side currents and possibly even lower than much of the dayside currents (except in the discontinuity region). However, the FUV profiles should be handled with caution because they can be affected by hydrocarbon absorption at altitudes below the methane homopause. Our simulations show that an emission profile peaking at $250 \mathrm{~km}$, as observed by Galileo-SSI, could lead to an apparent FUV profile very similar to an emission profile peaking at $400 \mathrm{~km}$. Moreover, a detailed analysis of the FUV spectra from the STIS instrument shows that the generally weak color ratio observed at the limb results from geometrical effects rather than from a weak absorption. The assessment of the shift between the peak altitudes of the emissions in the 123-130 nm range (i.e., absorbed by $\mathrm{CH}_{4}$ ) and in the $155-162 \mathrm{~nm}$ range (i.e., not absorbed by $\mathrm{CH}_{4}$ ) is a much more reliable way to detect absorption signatures above the limb and provides evidence for significant absorption compatible with a peak altitude of around $250 \mathrm{~km}$. Unfortunately, this observation does not rule out every ambiguity, as there is no certainty that the spectra in our data set capture the emission above the limb at the exact location where the main emission intersects the limb plane. Emissions taking place at a high altitude but beyond the limb rather than at the limb would still show some absorption signature because the line of sight would intersect the limb plane at a lower altitude.

In conclusion, our measurements provide a maximum value for the FUV peak altitude of the main emission on the nightside $(400 \mathrm{~km})$. However, a careful analysis of the spectral absorption signatures shows that these measurements are not incompatible with earlier measurements $(250 \mathrm{~km})$ and calls for systematic spectral observations along the limb with a proper slit orientation.

Acknowledgements. B. Bonfond is funded by the Fund for Scientific Research (F.R.S-FNRS). B. Bonfond, J. Gustin, D. Grodent, A. Radioti, and J.-C. Gérard are supported by the PRODEX program managed by ESA in collaboration with the Belgian Federal Science Policy Office. C. Tao was supported by a JSPS (Japan Society for the Promotion of Science) Postdoctoral Fellowships for Research Abroad. This research is based on observations with the NASA/ESA Hubble Space Telescope, obtained at the Space Telescope Science Institute, which is operated by AURA for NASA. It is based on publicly available observations acquired with the NASA/ESA Hubble Space Telescope (program IDs 7308, 7769, $8171,8657,10140,10507,10862,13035)$ and obtained from the Space Telescope Science Institute (https://archive.stsci.edu/hst/ search.php).

The topical editor E. Roussos thanks two anonymous referees for help in evaluating this paper.

\section{References}

Boffi, F. R., Sirianni, M., Lucas, R. A., Walborn, N. R., and Proffitt, C. R.: Delivery of a new ACS SBC throughput curve for Synphot, Technical Instrument Report ACS 2008-002, STSCI, tIR ACS 2008-002, 2008.

Bonfond, B., Grodent, D., Gérard, J.-C., Radioti, A., Dols, V., Delamere, P. A., and Clarke, J. T.: The Io UV footprint: Location, inter-spot distances and tail vertical extent, J. Geophys. Res., 114, A07224, doi:10.1029/2009JA014312, 2009.

Bonfond, B., Grodent, D., Gérard, J.-C., Stallard, T., Clarke, J. T., Yoneda, M., Radioti, A., and Gustin, J.: Auroral evidence of Io's control over the magnetosphere of Jupiter, Geophys. Res. Lett., 39, L01105, doi:10.1029/2011GL050253, 2012.

Bonfond, B., Gustin, J., Gérard, J. C., Grodent, D., Radioti, A., Palmaerts, B., Badman, S. V., Khurana, K. K., and Tao, C.: The far-yltraviolet main auroral emission at Jupiter - Part 1: Dawndusk brightness asymmetries, Ann. Geophys., 33, 1203-1209, doi:10.5194/angeo-33-1203-2015, 2015.

Cohen, I. J. and Clarke, J. T.: Modeling of Jupiter's auroral curtain and upper atmospheric thermal structure, J. Geophys. Res.Space, 116, A08205, doi:10.1029/2010JA016037, 2011.

Cravens, T. E.: Vibrationally excited molecular hydrogen in the upper atmosphere of Jupiter, J. Geophys. Res., 92, 11083-11100, doi:10.1029/JA092iA10p11083, 1987.

Delamere, P. A., Bagenal, F., Paranicas, C., Masters, A., Radioti, A., Bonfond, B., Ray, L., Jia, X., Nichols, J., and Arridge, C.: Solar Wind and Internally Driven Dynamics: Influences on Magnetodiscs and Auroral Responses, Space Sci. Rev., 187, 51-97, doi:10.1007/s11214-014-0075-1, 2014.

Gérard, J.-C., Bonfond, B., Gustin, J., Grodent, D., Clarke, J. T., Bisikalo, D., and Shematovich, V.: Altitude of Saturn's aurora and its implications for the characteristic energy of precipitated electrons, Geophys. Res. Lett., 36, L02202, doi:10.1029/2008GL036554, 2009.

Gérard, J.-C., Bonfond, B., Grodent, D., Radioti, A., Clarke, J. T., Gladstone, G. R., Waite, J. H., Bisikalo, D., and Shematovich, V. I.: Mapping the electron energy in Jupiter's aurora: Hubble spectral observations, J. Geophys. Res.-Space, 119, 9072-9088, doi:10.1002/2014JA020514, 2014.

Grodent, D.: A Brief Review of Ultraviolet Auroral Emissions on Giant Planets, Space Sci. Rev., 187, 23-50, doi:10.1007/s11214014-0052-8, 2015.

Grodent, D., Waite, J., and Gérard, J.-C.: A self-consistent model of the Jovian auroral thermal structure, J. Geophys. Res., 106, 12933-12952, doi:10.1029/2000JA900129, 2001.

Grodent, D., Clarke, J. T., Kim, J., Waite, J. H., and Cowley, S. W. H.: Jupiter's main auroral oval observed with HST-STIS, J. Geophys. Res., 108, 1389, doi:10.1029/2003JA009921, 2003.

Grodent, D., Bonfond, B., Gérard, J.-C., Radioti, A., Gustin, J., Clarke, J. T., Nichols, J., and Connerney, J. E. P.: Auroral evidence of a localized magnetic anomaly in Jupiter's northern hemisphere, J. Geophys. Res., 113, A09201, doi:10.1029/2008JA013185, 2008.

Gustin, J., Gérard, J.-C., Grodent, D., Cowley, S. W. H., Clarke, J. T., and Grard, A.: Energy-flux relationship in the FUV Jovian aurora deduced from HST-STIS spectral observations, J. Geophys. Res., 109, A10205, doi:10.1029/2003JA010365, 2004.

Gustin, J., Cowley, S. W. H., Gérard, J.-C., Gladstone, G. R., Grodent, D., and Clarke, J. T.: Characteristics of Jo- 
vian morning bright FUV aurora from Hubble Space Telescope/Space Telescope Imaging Spectrograph imaging and spectral observations, J. Geophys. Res.-Space, 111, A09220, doi:10.1029/2006JA011730, 2006.

Hess, S., Bonfond, B., Chantry, V., Gérard, J.-C., Grodent, D., Jacobsen, S., and Radioti, A.: Evolution of the Io footprint brightness II: Modeling, Planet. Space Sci., 88, 76-85, doi:10.1016/j.pss.2013.08.005, 2013.

James, G. K., Ajello, J. M., and Pryor, W. R.: The middle ultraviolet-visible spectrum of $\mathrm{H}_{2}$ excited by electron impact, J. Geophys. Res., 103, 20113-20124, doi:10.1029/97JE03692, 1998.

Knight, S.: Parallel electric fields, Planet. Space Sci., 21, 741-750, doi:10.1016/0032-0633(73)90093-7, 1973.

Lundin, R. and Sandahl, I.: Some characteristics of the parallel electric field acceleration of electrons over discrete auroral arcs as observed from two rocket flights, in: European Sounding Rocket, Balloon and Related Research, with Emphasis on Experiments at High Latitudes, edited by: Halvorsen, T. and Battrick, B., ESA Special Publication, 135, 125-136, 1978.

Nichols, J. D., Bunce, E. J., Clarke, J. T., Cowley, S. W. H., Gérard, J.-C., Grodent, D., and Pryor, W. R.: Response of Jupiter's UV auroras to interplanetary conditions as observed by the Hubble Space Telescope during the Cassini flyby campaign, J. Geophys. Res.-Space, 112, A02203, doi:10.1029/2006JA012005, 2007.

Nichols, J. D., Clarke, J. T., Gérard, J. C., Grodent, D., and Hansen, K. C.: Variation of different components of Jupiter's auroral emission, J. Geophys. Res.-Space, 114, A06210, doi:10.1029/2009JA014051, 2009.

Palmaerts, B., Radioti, A., Grodent, D., Chané, E., and Bonfond, B.: Transient small-scale structure in the main auroral emission at Jupiter, J. Geophys. Res. Space Physics, 119, 9931-9938, doi:10.1002/2014JA020688, 2015.
Radioti, A., Gérard, J.-C., Grodent, D., Bonfond, B., Krupp, N., and Woch, J.: Discontinuity in Jupiter's main auroral oval, J. Geophys. Res., 113, A01215, doi:10.1029/2007JA012610, 2008.

Ray, L. C., Ergun, R. E., Delamere, P. A., and Bagenal, F.: Magnetosphere-ionosphere coupling at Jupiter: A parameter space study, J. Geophys. Res.-Space, 117, A01205, doi:10.1029/2011JA016899, 2012.

Shematovich, V. I., Bisikalo, D. V., Gérard, J.-C., Cox, C., Bougher, S. W., and Leblanc, F.: Monte Carlo model of electron transport for the calculation of Mars dayglow emissions, J. Geophys. Res., 113, E02011, doi:10.1029/2007JE002938, 2008.

Uno, T., Kasaba, Y., Tao, C., Sakanoi, T., Kagitani, M., Fujisawa, S., Kita, H., and Badman, S. V.: Vertical emissivity profiles of Jupiter's northern $\mathrm{H}_{3}^{+}$and $\mathrm{H}_{2}$ infrared auroras observed by Subaru/IRCS, J. Geophys. Res.-Space, 119, 10219, doi:10.1002/2014JA020454, 2014.

Vasavada, A. R., Bouchez, A. H., Ingersoll, A. P., Little, B., Anger, C. D., and The Galileo SSI Team: Jupiter's visible aurora and Io footprint, J. Geophys. Res., 104, 27133-27142, 1999.

Vogt, M. F., Kivelson, M. G., Khurana, K. K., Walker, R. J., Bonfond, B., Grodent, D., and Radioti, A.: Improved mapping of Jupiter's auroral features to magnetospheric sources, J. Geophys. Res.-Space, 116, A03220, doi:10.1029/2010JA016148, 2011.

Yung, Y. L., Gladstone, G. R., Chang, K. M., Ajello, J. M., and Srivastava, S. K.: $\mathrm{H}_{2}$ fluorescence spectrum from 1200 to $1700 \mathrm{~A}$ by electron impact - Laboratory study and application to Jovian aurora, Astrophys. J., 254, L65-L69, doi:10.1086/183757, 1982. 